\title{
ELECTRON IDENTIFICATION USING A SYNCHROTRON RADIATION DETECTOR
}

\author{
J.S. DWORKIN *, P.T. COX **, E.C. DUKES *** and O.E. OVERSETH \\ Department of Physics, University of Michigan, Ann Arbor, Michigan 48109, USA
}

\author{
R. HANDLER, R. GROBEL ${ }^{+}$, A. JASKE, B. LUNDBERG ${ }^{++}$, G. OTT, L. PONDROM, M. SHEAFF \\ and C. WILKINSON
}

Physics Department, University of Wisconsin, Madison, Wisconsin 53706, USA

\author{
K. HELLER \\ School of Physics and Astronomy, University of Minnesota, Minneapolis, Minnesota 55455, USA
}

\author{
A. BERETVAS, L. DECK ${ }^{+++}$, T. DEVLIN, K.B. LUK ${ }^{\S}$ and R. RAMEIKA $\$$ \\ Physics Department, Rutgers-The State University, P.O Box 849. Piscataway, New Jersey 08854, USA
}

Received 20 November 1985

A xenon filled multuwire proportional chamber was used to detect synchrotron radiation from high energy electrons traversing the field of a standard spectrometer magnet. Signals from the chamber were used to achieve an electron trigger with a pion rejection of -17 and an average electron detection efficiency of $81 \%$. Off-line analysis of the chamber signals increased the pion rejection to 59 with an electron efficiency of $77 \%$.

\section{Introduction}

The possibility of identifying electrons by detecting their synchrotron radiation has been considered previously [1,2]. Merkel et al. [3] observed synchrotron radiation from electrons with a xenon-filled multiwire proportional chamber (MWPC) designed to detect transition radiation $\mathrm{X}$-rays. In this paper we describe the performance of such a detector used to identify electrons in the momentum range $6-34 \mathrm{GeV} / c$. The detector was operated as part of the lambda beta decay

* Present address: Fonar Corporation, 110 Marcus Drive, Melville, NY 11747, USA.

** Present address: Physics Department, Rockefeller University, New York, NY 10021, USA.

*** Present address: CERN, CH-1211, Geneva 23, Switzerland.

+ Present address: Salomon Brothers, Inc., One New York Plaza, New York, NY 10004, USA.

${ }^{++}$Present address: Physics Department, Ohio State University, Columbus, $\mathrm{OH} 43210$, USA.

${ }^{+++}$Present address: Honeywell, Inc., 10400 Yellow Circle Drive, Minnetonka, MN 55343, USA.

$\S$ Present address: Fermilab, P.O. Box 500, Batavia, IL 60510, USA. experiment [4] performed in the Fermilab neutral-hyperon beam [5].

The detector design was motivated by the key experimental problem in the lambda beta decay experiment, namely, distinguishing between electrons from the rare decay $\Lambda \rightarrow \operatorname{pe} \bar{\nu}$ and pions from the roughly 1000 times more frequent decay $\Lambda \rightarrow \mathrm{p} \pi^{-}$. In our experiment the electrons traversed a uniform magnetic field used to analyze the momentum of charged decay products. The resulting acceleration of the electron caused synchrotron radiation to be emitted in the $\mathrm{X}$-ray region. In contrast, the more massive pions and protons, passing through the same magnetic field, emitted a negligible amount of synchrotron radiation. A xenon-filled MWPC located immediately downstream of the analysis magnet, detected the photoelectrons ejected from xenon atoms by the incident $\mathrm{X}$-ray photons. The electrons were identified by the characteristic patterns of synchrotron radiation emitted in the analysis magnet. Any charged particle passing through the chamber was also detected. A pion rejection of $98 \%$ was achieved with an electron detection efficiency of $77 \%$. More stringent criteria on the synchrotron radiation pattern increased the pion rejection to $99.7 \%$ but reduced the electron efficiency to $53 \%$. 
The design of the xenon chamber is described in sect. 2 followed by a review of the relevant characteristics of both synchrotron radiation and the photoelectric absorption process. Sect. 3 presents the electron identification efficiency and pion rejection factor achieved with the detector. Finally, we discuss the design and performance of an electronic trigger processor which provided online electron-pion discrimination by examining the data from the synchrotron radiation detector.

\section{The synchrotron radiation detector}

\subsection{Xenon chamber design}

The construction of the xenon chamber, illustrated in fig. 1, was similar to that of our standard MWPC [6]. The five planes between the entrance and exit windows contained wires soldered to printed circuit boards which had been epoxied to G-10 fiberglass frames. The interplane spacing was $4.8 \mathrm{~mm}$ giving an active path length of $19.2 \mathrm{~mm}$ between the entrance and exit cathode planes. The three cathode planes were constructed with $64 \mu \mathrm{m}$ diameter $\mathrm{Be}-\mathrm{Cu}$ wires. These wires were placed $1.0 \mathrm{~mm}$ apart in the vertical entrance and exit cathode planes. The third cathode plane, consisting of a 192 wire horizontal wires with $1.33 \mathrm{~mm}$ wire spacing, was placed between the two vertical sense planes. Each sense plane contained $32025 \mu \mathrm{m}$ diameter gold-plated tungsten wires, spaced $2 \mathrm{~mm}$ apart. Signals were detected from both sense planes and the central cathode plane. The xenon gas filling the chamber was first passed at atmospheric pressure through liquid methylal at $0^{\circ} \mathrm{C}$.

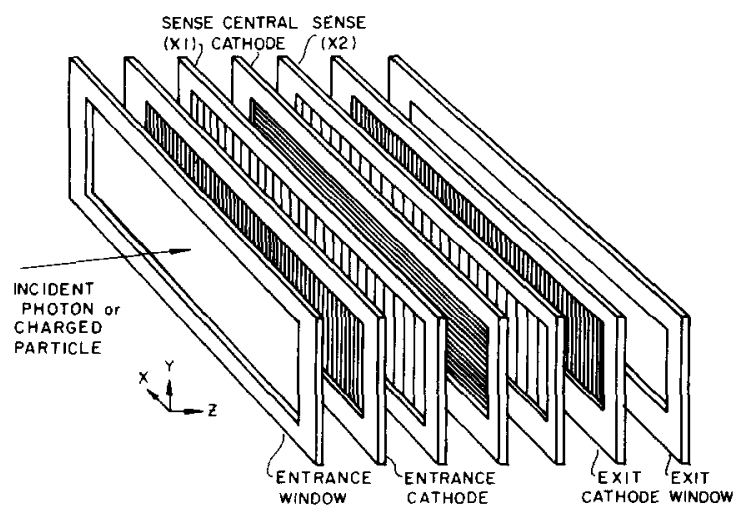

Fig. 1. The construction of the xenon chamber. The active region between the entrance and exit cathode planes contained $19.2 \mathrm{~mm}$ of xenon. The two vertical sense planes and the horizontal central cathode plane detected the photoelectric conversions of incident $\mathrm{X}$-ray photons as well as incident charged particles passing through the chamber.

\subsection{Synchrotron radiation and photoelectric absorption}

The synchrotron radiation emitted by a charged particle bending in a magnetic field is characterized by the total emitted energy, $I$, and the critical photon energy, $\hbar \omega_{c}$, below which a majority of the radiation is emitted [7]. With the charged particle mass $(M)$ and energy $(E)$ in $\mathrm{GeV}$, the magnetic field strength $(B)$ in $\mathrm{T}$ and the path length $(L)$ in $\mathrm{m}$, these quantities are

$$
\begin{aligned}
& I=\left(8.59 \times 10^{-14}\right) \frac{E^{2} B^{2} L}{M^{4}} \quad[\mathrm{keV}], \\
& I=1.27 E^{2} B^{2} L \quad[\mathrm{keV}] \quad \text { (for electrons) } \\
& \hbar \omega_{\mathrm{c}}=\left(1.77 \times 10^{-10}\right) \frac{E^{2} B}{M^{3}} \quad[\mathrm{keV}], \\
& \hbar \omega_{\mathrm{c}}=1.33 E^{2} B \quad[\mathrm{keV}] \quad \text { (for electrons). }
\end{aligned}
$$

For a charged pion the total energy radiated is approximately $10^{-10}$ that of an electron of equal momentum.

The number of photons emitted can be estimated by assuming the average photon energy is $\hbar \omega_{\mathrm{c}} / 4$ :

$n_{\gamma}=4 I / \hbar \omega_{\mathrm{c}}$,

$n_{\gamma}=3.8 B L \quad$ (for electrons).

The constant field integral in the analysis magnet was $3.13 \mathrm{~T} \mathrm{~m}(B=1.65 \mathrm{~T}, L=1.9 \mathrm{~m})$ so approximately 12 photons were emitted by each electron independent of its energy. As an example, a $10 \mathrm{GeV}$ electron radiated a total of $657 \mathrm{keV}$ as it was bent in the analysis magnet, and the average photon energy was $56 \mathrm{keV}$, an X-ray.

The properties of the detector depend on the channels available in the photoelectric process for an incident $\mathrm{X}$-ray photon [8]. With its $\mathrm{K}$ and L-shell edges at 34.6 and $4.8 \mathrm{keV}$, xenon is suited for detecting incident $\mathrm{X}$-ray photons from several $\mathrm{keV}$ to over $100 \mathrm{keV}$. Argon, normally the main component of the gas mixture in multiwire proportional chambers, has a photoelectric absorption mean free path for X-rays in this energy range which is generally about an order of magnitude higher than for xenon [9]. In our detector, which had a xenon thickness of $1.9 \mathrm{~cm}$, a $50 \mathrm{keV}$ photon $(7.6 \mathrm{~cm}$ mean free path) had a photoelectric absorption probability of $22 \%$ while a $5 \mathrm{keV}$ photon $(2.7 \mathrm{~mm}$ mean free path) had an absorption probability of $99.9 \%$. The more energetic the electron the higher the average photon energy, and the less the photoelectric absorption probability. Furthermore, the probability that the chamber gave a detectable signal also depended on the range and thus the energy of the ejected photoelectron. Typical values varied from $0.3 \mathrm{~mm}$ for $6 \mathrm{keV}$ photoelectrons to more than $6 \mathrm{~mm}$ for photoelectrons with energies greater than $40 \mathrm{keV}$. The number of $\mathrm{X}$-rays actually detected from $8 \mathrm{GeV}$ electrons is compared to the number from 


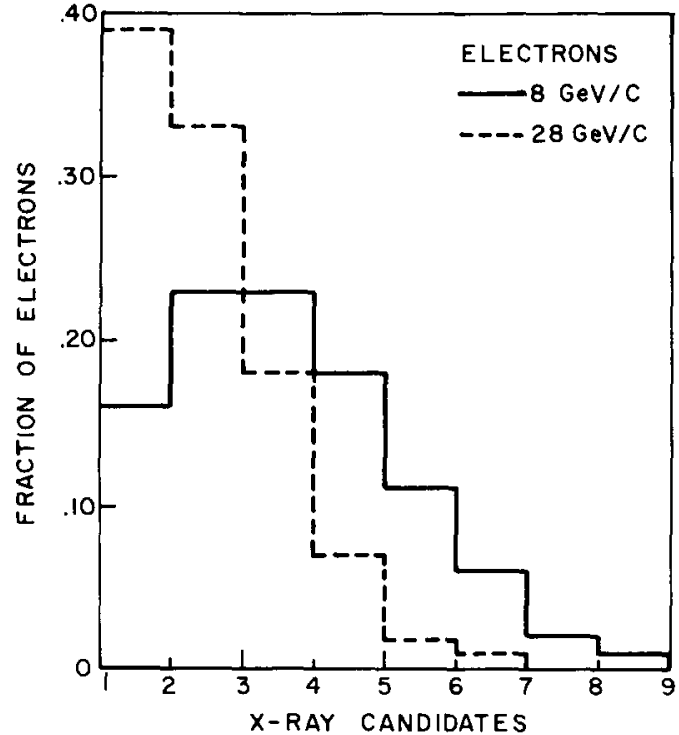

Fig 2. The number of $X$-ray candidates for 8 and $28 \mathrm{GeV} / c$ electrons satisfying the least restrictive electron signature as described in the text.
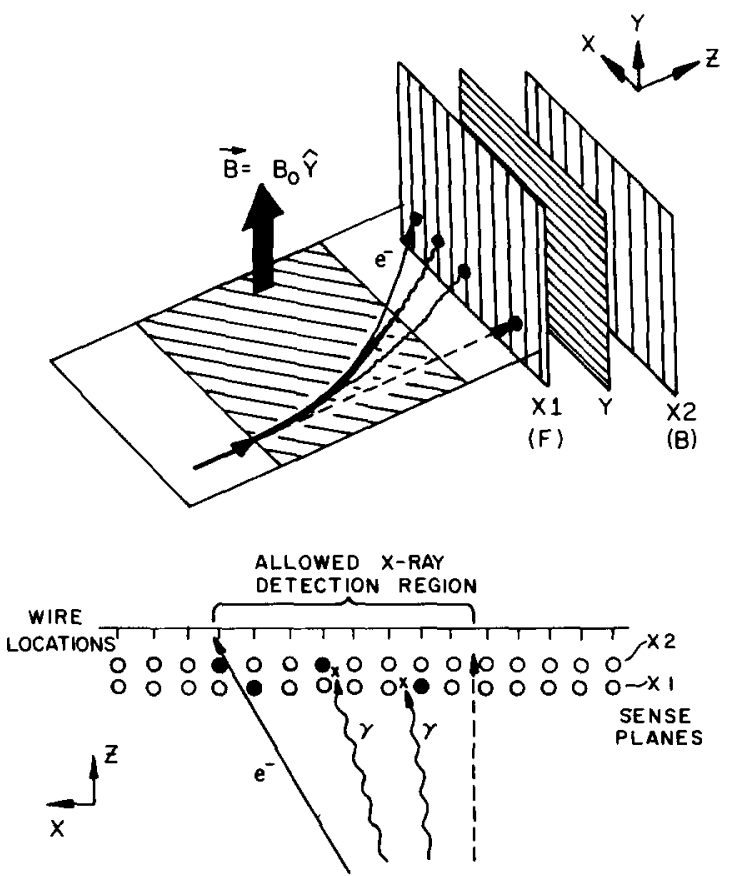

Fig. 3. (a) The synchrotron X-rays from an electron arrived at the same location in the central cathode plane of the xenon chamber as the deflected electron passing through the chamber. The wires in the two vertical sense planes (X1, X2) were parallel to the analysis magnetic field bending the electron. (b) A typical sense plane pattern from an electron with two detected $\mathrm{X}$-ray photons. The allowed $\mathrm{X}$-ray detection region corresponding to the electron trajectory is shown.
$28 \mathrm{GeV}$ electrons in fig. 2 illustrating this expected decrease as the incident electron momentum increased.

\subsection{An electron signature}

A typical signature for an electron radiating two $\mathrm{X}$-ray photons is shown in fig. 3. The $\mathrm{X}$-ray photons are emitted in the plane of the electron orbit in an extremely narrow cone of half-angle $m_{\mathrm{e}} / E_{\mathrm{e}}$ about the electron direction. In our experiment, the electron remained undeflected in the vertical $(y)$ direction so the photons intersected the xenon chamber at the same wire location in the central cathode plane as the incident electron. In the sense planes, the orientation of the wires was chosen parallel to the field in the analysis magnet. The photons arrived at a region bounded by the intersection with the tangent to the electron track at the entrance of the analysis magnet, and the location of the deflected electron as it passed through the chamber. The presence of at least one photon detected in this allowed region distinguished an electron from a charged pion. A short range photoelectron resulting from the incident $\mathrm{X}$-ray photon interacting in xenon produced a signal on a wire in only one of the sense planes. In contrast, an incident charged particle passing through the chamber gave a signal in both sense planes.

\section{Detector performance}

\subsection{Experimental arrangement}

Fig. 4 shows the plan and elevation views of the apparatus used for the lambda beta decay experiment. The salient features will be reviewed here. The neutralhyperon beam contained neutral strange particles, along with a factor of 100 higher flux of neutrons and photons. Lambda hyperons were detected by observing their charged decay products in the magnetic spectrometer downstream of the evacuated decay region. The characteristic neutral vee decay topology, consisting of two oppositely charged particles emanating from a single point in the decay region, is illustrated for the case of the rare beta decay $\Lambda \rightarrow \mathrm{pe} \bar{\nu}$. This signature was also typical of the more copious nonleptonic decay $\Lambda \rightarrow \mathrm{p} \pi^{-}$. Electron-positron pairs produced by photons from the neutral beam interacting in the small amount of material upstream of the first MWPC resulted in a similar topology. These events were distinguished by a small laboratory opening angle between the electron and the positron.

The magnetic spectrometer included two sets of three argon-filled MWPCs $(\mathrm{C} 1-\mathrm{C} 6)$ which measured the track coordinates of the charged decay products upstream and downstream of the dipole analysis magnet. This homogeneous vertical $(+y)$ magnetic field deflected 


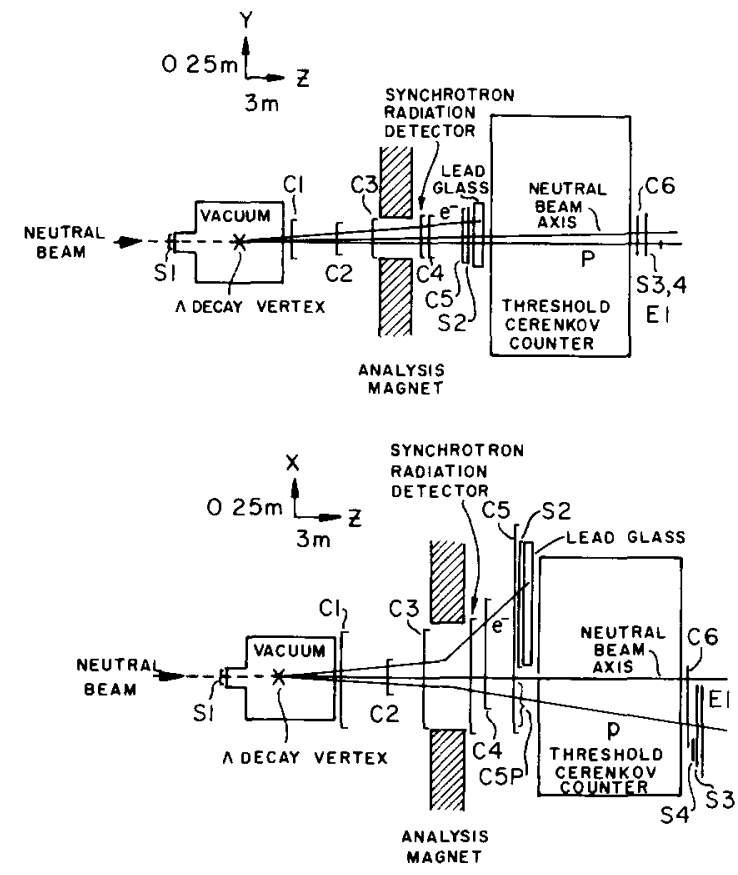

Fig. 4. The plan and elevation views of the experimental apparatus used for the lambda beta decay experıment. The synchrotron radiation detector was located immediately downstream of the analysis magnet. The S's denote scintillation counters and the C's refer to the argon-filled multiwire proportional chambers in the magnetic spectrometer. A characteristic neutral vee decay topology for $\Lambda \rightarrow$ pe $\tilde{\nu}$ is illustrated.

negatively charged particles in the horizontal $(+x)$ direction. The synchrotron radiation detector completely covered the downstream aperture of the magnet.

Electron identification was provided by the xenon chamber and by an array of SF2 lead glass blocks (32 $\mathrm{mm}$ radiation length) located downstream of the xenon chamber. The lead glass array consisted of 64 blocks each $10 \mathrm{~cm} \times 10 \mathrm{~cm} \times 39 \mathrm{~cm}$. The array was 8 blocks wide (along the $X$ axis) and 8 blocks deep (along $Z$ ). The largest dimension of each block was vertical $(Y)$. Electron identification depended on the shape of the shower development through the 8 block deep array as well as the total pulse height [4].

\subsection{Xenon chamber operation}

The cathode planes were held at a potential of $-3500 \mathrm{~V}$. The chamber's operation was monitored by observing the output from an emitter-follower driven by a selected sense wire (outside the active area) which detected the ionization from $5.9 \mathrm{keV}$ X-rays emitted by an attached ${ }^{55} \mathrm{Fe}$ source. Every sense wire and each group of three adjacent central cathode plane wires were connected to an amplifier channel which triggered a one-shot circuit when the detected signal exceeded a threshold of $0.8 \mathrm{mV}$.

The scheme to record data from the active amplifier channels in the xenon chamber electronics was similar to that used for the other MWPCs $(\mathrm{C} 1-\mathrm{C} 6)$. The edges of the one-shots were first differentiated. Latches corresponding to the active amplifier channels were set when the differentiated pulse from the trailing edge of the one shot coincided with the arrival of a gate pulse generated by the fast trigger logic. The width of the gate pulse sent to the xenon chamber electronics was 150 ns. This allowed ample time for the pulse originated by a short range photoelectron ejected from a xenon atom to reach a wire in the xenon chamber.

In order to reduce any $\mathrm{X}$-ray absorption upstream of the xenon chamber, a helium-filled polyethylene bag was inserted in the gap of the analysis magnet. The downstream end of the bag was connected to the chamber's upstream fiberglass frame. Two $25-\mu \mathrm{m}$ thick mylar sheets separated by $5 \mathrm{~mm}$ of air comprised a barrier between the helium in the bag and the gas mixture in the chamber. The helium pushed this window towards the entrance cathode plane, minimizing the amount of xenon in the region upstream of the active volume.

\subsection{Callbratıon data}

The electron identification efficiency and the charged pion rejection of the xenon chamber were studied with momentum-analyzed electrons and charged pions obtained with special calibration triggers. By constructing a logical OR of these triggers, suitably prescaled, with the $\beta$ decay trigger, $\mathrm{e}^{+} \mathrm{e}^{-}$pairs, $\Lambda \rightarrow \mathrm{p} \pi^{-}$events and $\Lambda \rightarrow$ pe $\bar{\nu}$ events were accumulated simultaneously.

The results quoted below are based on the analysis of $142000 \Lambda \rightarrow \mathrm{p} \pi^{-}$events and $44000 \mathrm{e}^{+} \mathrm{e}^{-}$pairs. These events were selected by applying the usual reconstructed mass and topology constraints. Additional $\mathrm{e}^{-}-\pi^{-}$discrimination was provided by the lead glass array. Positrons were identified by a threshold Cherenkov counter and a simple shower counter (E1) consisting of 2 radiation lengths of lead followed by a plastic scintillation counter. Calibration event selection criteria required that the electrons from pairs and pions from $\Lambda \rightarrow \mathrm{p} \pi^{-}$had comparable allowed synchrotron radiation regions in the xenon chamber. Events with any detected synchrotron radiation which could have been emitted by the positive particle were rejected. Both the pion contamination of the electron sample and the electron contamination of the pion sample were less than $0.1 \%$.

\subsection{Xenon chamber electron identification and plon rejec- tion}

The least stringent xenon chamber electron signature demanded the detection of at least one $\mathrm{X}$-ray candidate 
in either sense plane as shown in fig. 3. As expected the identification efficiency decreased with electron momentum dropping from $93 \%$ for $8 \mathrm{GeV} / c$ electrons to $74 \%$ for $32 \mathrm{GeV} / c$ electrons as shown in fig. 5 . For a flat momentum spectrum from $6-34 \mathrm{GeV} / c$, the average electron identification efficiency was found to be $84 \%$. The corresponding pion rejection factor, shown in fig. 6 , was 44 , essentially independent of momentum.

A more restrictive electron signature increased the average pion rejection factor to 320 , but decreased the average electron efficiency to $53 \%$ as is also shown in figs, 5 and 6 . The additional requirements were developed by studying the xenon chamber patterns for the $\Lambda \rightarrow \mathrm{p} \pi^{-}$events where any X-ray candidates are spurious. The main sources of these candidates were energetic knock-on electrons, accidental neutral beam interactions and accidental charged particles passing through the xenon chamber. To increase the rejection, the sense planes were required to contain only X-ray candidates in addition to the expected hits from the charged particle tracks.

The sense plane detection efficiency for charged particles was measured to be $98-100 \%$. The efficiency of the central cathode plane was $90 \%$ for pions and $97 \%$ for electrons.

Events with additional chamber hits outside the negatively charged particle's allowed X-ray detection region were rejected. The $\mathrm{X}$-ray candidates and charged particle candidates were also required to contain less than four adjacent wires hit. An important additional constraint for the electron signature was the require-

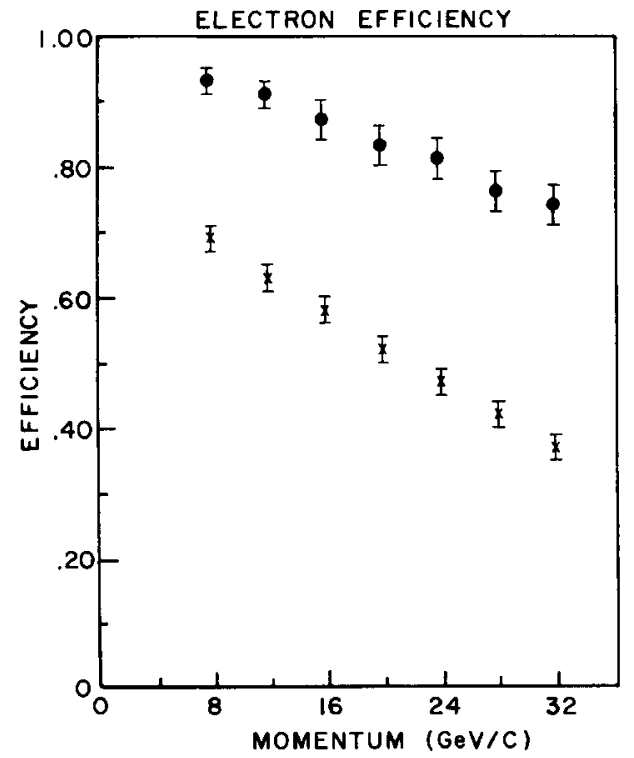

Fig. 5. The measured electron identification efficiency for the least restrictive electron signature, solid dots, and the more restrictive electron signature, crosses, described in the text.

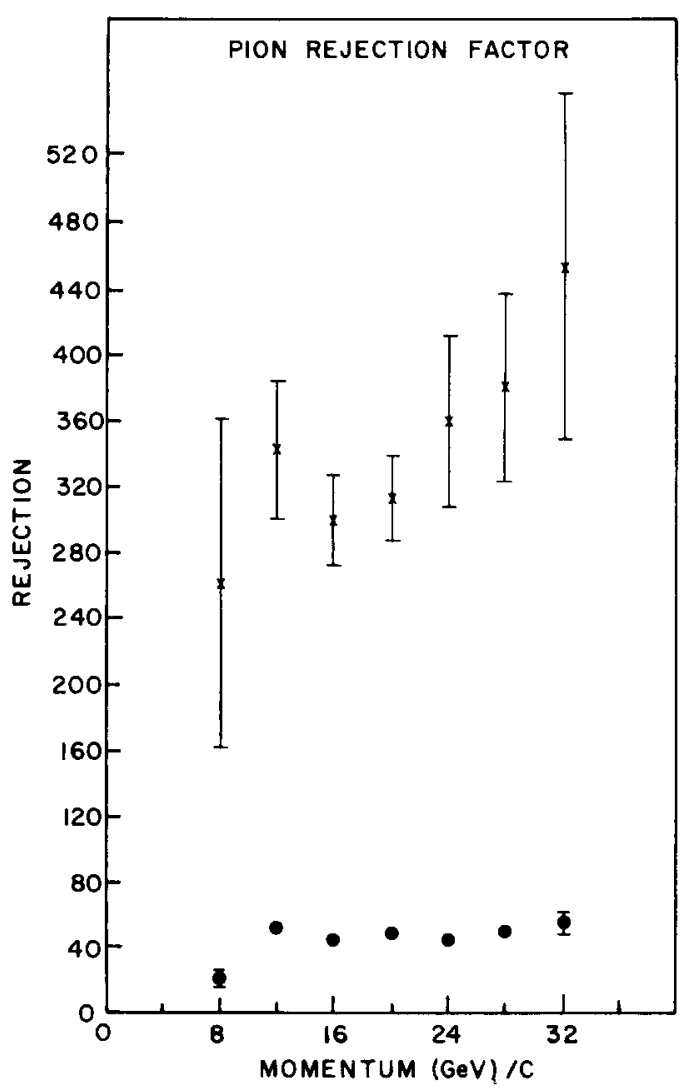

Fig. 6. The measured pion rejection factor for the least restrictive electron signature and the more restrictive electron signature described in the text.

ment of coplanarity of the electron and the X-ray candidates. Thus any event which had hits in the central cathode plane in addition to those associated with the charged particles was rejected.

\section{The electron trigger processor}

\subsection{Design considerations and efficiency}

On-line electron-pion discrimination was provided by an ECL processor which, in $16 \mu \mathrm{s}$, examined the data from the xenon chamber and triggered the data acquisition system on two-track events containing an electron. The synchrotron radiation trigger (SR) was based on the photoelectric conversion hit pattern which gives a wire hit in just one of the sense planes. A charged particle passing through the chamber causes an active wire in both sense planes. The processor compared the data from the two sense planes, at each of the 320 wire locations. The on-line electron trigger efficiency, shown in fig. 7, was measured by recording the 


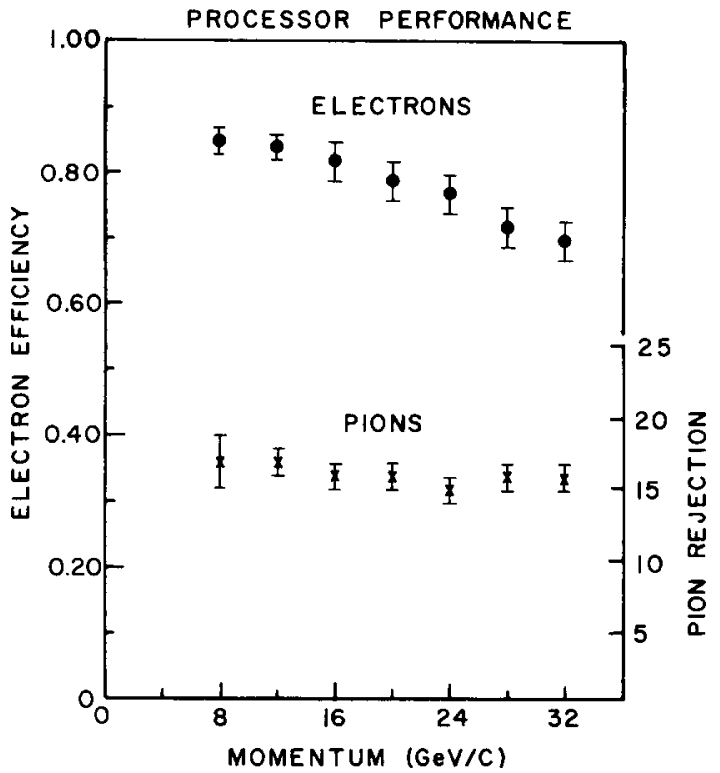

Fig. 7. The on-line electron identification efficiency and pion rejection factor achieved with the trigger processor.

outcome of the trigger decision in a coincidence register for every calibration event. For a flat momentum spectrum extending from $6-34 \mathrm{GeV} / c$, the on-line electron trigger efficiency was measured to be $81 \%$ with an average pion rejection factor of 17 .

\subsection{Processor operation and trigger requirements}

A pretrigger, signifying that an event had the proper topology, initiated the processor operation which is outlined schematically in fig. 8 . In our experiment the pretrigger rate was about $600 \mathrm{~s}^{-1}$ for $5 \times 10^{9}$ protons/s on target. The decisions for a typical $\mathrm{e}^{+} \mathrm{e}^{-}$pair and a $\Lambda \rightarrow \mathrm{p} \pi^{-}$event are illustrated in fig. 9. The wire hit data was latched by the pretrigger and then read from both sense planes (X1, X2) simultaneously. A $20 \mathrm{MHz}$ clock caused the two shift registers to sequentially strobe wire hit information on data lines. These data pulses were shaped so that the wire hit pattern for each plane was encoded in a string of $20 \mathrm{~ns}$ pulses (DX1, DX2) with a pulse spacing of $50 \mathrm{~ns}$.

In the decision area, the pulses were stretched (STR1, STR2) to $80 \mathrm{~ns}$. Since a stretched pulse overlapped the pulse corresponding to a wire hit at the next location to be examined, contiguous hits in a given sense plane were represented by a single wide pulse. This is illustrated in fig. 9a for the photon conversion occurring in the X1 sense plane. Counters, denoted $B(O)$ and $B(A)$, were incremented by the logical ORs, $B(O)$, and logical ANDs, $B(A)$, between the two sets of pulses representing the clustered hits in the sense planes.

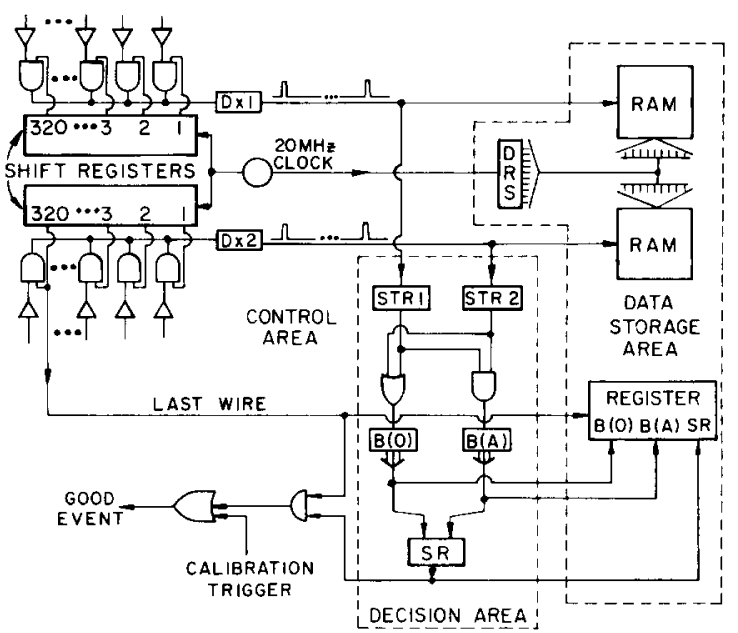

Fig. 8. A schematic illustration of the trigger processor operation. Strings of $20 \mathrm{~ns}$ pulses representing the spatial location of data in each of the two sense planes (DX1, DX2) were compared in the decision area to decide if synchrotron radiation (SR) had been detected in a two-track event. The identity of the wire hits were encoded in the data storage area.

After all of the 320 latches for each sense plane had been strobed, the synchrotron radiation trigger logic (SR) used the outputs of $B(O)$ and $B(A)$ to determine if synchrotron radiation had been detected.

A logical AND corresponded to an incident charged particle passing through the xenon chamber while a logical OR could be an X-ray candidate. The pulse stretching ensured that a comparison between wire hits at adjacent wire locations in different sense planes would also form a logical AND illustrated in fig. $9 \mathrm{~b}$. Since a logical AND also satisfies a logical OR, both $B(O)$ and $B(A)$ are incremented for a charged particle. Thus an event with only incident charged particles passing through the chamber resulted in the same number of ANDs and ORs. The $\Lambda \rightarrow \mathrm{p} \pi^{-}$event in fig. 9b provides an example $(B(O)=B(A)=2)$. An additional logical $O R$ was the signature of a synchrotron radiation candidate. The SR trigger logic required at least one such additional count in $B(O)$.

$\mathrm{SR}=([B(0)-B(A)] \geq 1)(B(O) \geq 3)(B(A) \leq 5)$.

The last requirement was used to remove multuple charged track events from consideration. The $\mathrm{e}^{+} \mathrm{e}^{-}$pair in fig. 9a satisfied all three requirements $(B(O)=$ 4, $B(A)=2$ ).

As the sense plane wire data were being compared in order to arrive at the SR decision, the wire hit positions were also encoded and stored in the data storage area. A nine-bit scaler (DRS) was incremented each time a different wire location was examined. A pulse on DX1 or DX2 indicated the presence of a wire hit at the most 

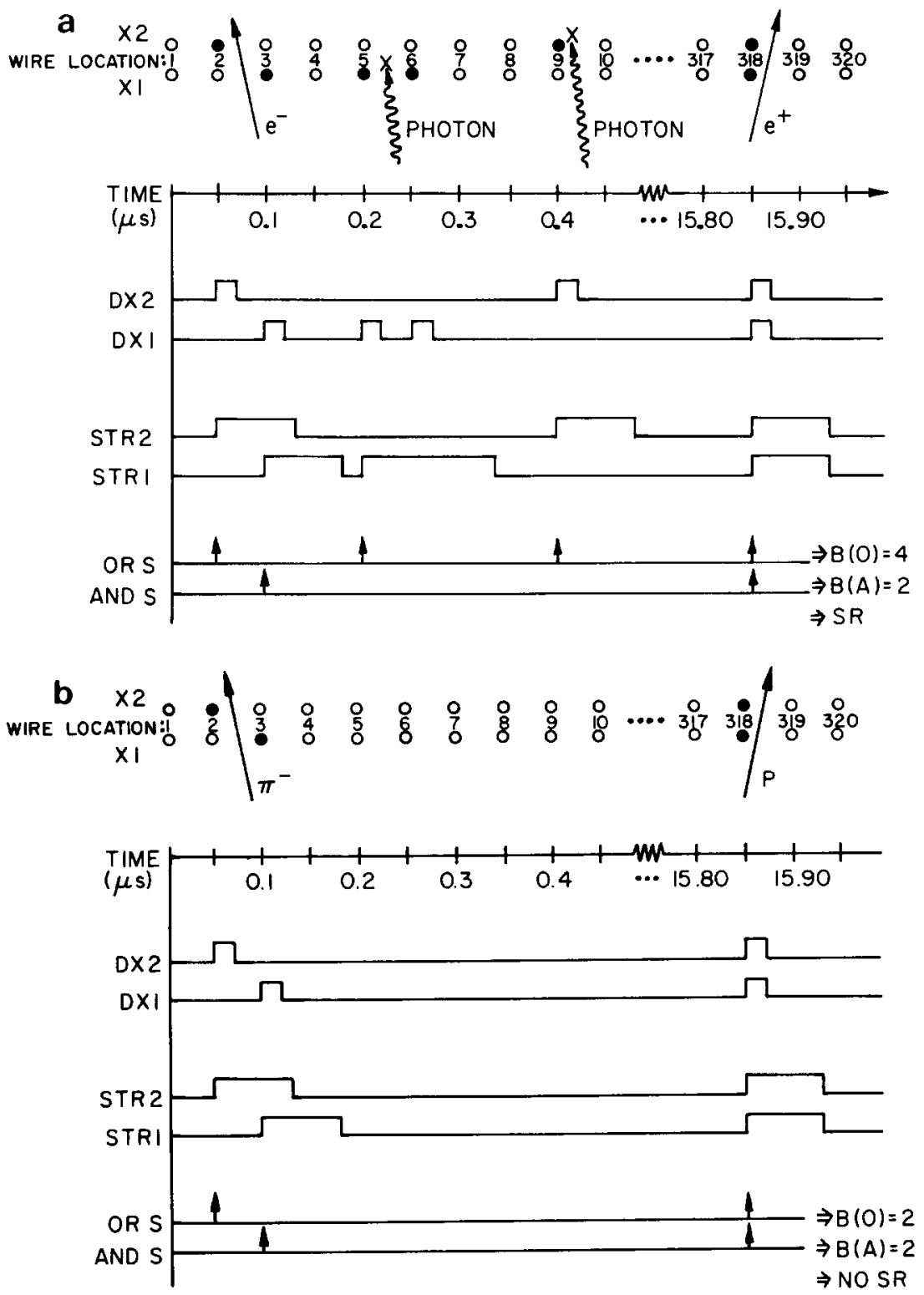

Fig. 9. The pulses generated by the processor corresponding to the sense plane wire hit patterns for: (a) a typical $\mathrm{e}^{+} \mathrm{e}^{-}$pair which satisfied the SR trigger logic; (b) a typical $\Lambda \rightarrow \mathrm{p} \pi^{-}$event falling to satisfy the SR trigger logic.

recently examined wire location and caused the binary contents of the DRS to be loaded into the appropriate $16 \times 16$ RAM. To monitor the operation of the processor, the binary outputs of $B(O)$ and $B(A)$ and the SR state were loaded into a register after all sense plane wires had been examined. For a successful beta decay trigger, the wire hit data stored in the RAMs and the contents of this register were then retrieved by the PDP11/CAMAC data acquisition system and written to tape.

\section{Conclusion}

For those experiments which already employ magnetic spectrometers to determine the momentum of high energy charged particles, synchrotron radiation is an excellent method of identifying electrons. Such radiation occurs in any case and only requires a suitable detector which will not interfere with other measurements. A xenon filled MWPC provides a good, low mass solution. The distinctive pattern of wire hits in the 
chamber provides a useful on-line signal for an experiment trigger which can be refined offline to give an excellent means of identifying high energy electrons [10]. For a flat momentum spectrum extending from 6-34 GeV/c, the combination of the on-line trigger, described in sect. 4.1 , with the least stringent offline electron signature, described in sect. 3.4, gave an overall electron identification efficiency of $77 \%$ an average pion rejection of 59.

\section{Acknowledgment}

This work was supported by the U.S. Department of Energy and the National Science Foundation. One of us (K.H.) would like to thank the University of Utah where some of this paper was written.

\section{References}

[1] L. Marshall, Rev. Sci. Instr. 30 (1959) 412.

[2] W. Busza et al., Nucl. Instr. and Meth. 105 (1972) 613.

[3] B. Merkel et al., Nucl. Instr. and Meth. 138 (1976) 625.

[4] For details see J. Dworkin, Ph.D. thesıs, University of Michigan (1983) unpublished.

[5] P. Skubic et al., Phys. Rev. D18 (1978) 3115; L. Pondrom. Phys. Rep. 122 (1985) 57.

[6] P. Skubic, Ph.D. Thesis, University of Michıgan (1977) unpublished.

[7] J.D. Jackson, Classical Electrodynamics (Wiley, New York, 1975).

[8] J.E. Batemen et al., Nucl. Instr. and Meth. 135 (1976) 235

[9] W.M.J. Veigele, Atomic Data Tables 5 (1973) 51; U. Gastaldi, Nucl. Instr. and Meth. 157 (1978) 441.

[10] A. Bodek et al., AMY: Proposal to TRISTAN, University of Rochester (1983) unpublished. 